\title{
Arterial Spin-Labeled Perfusion of Pediatric Brain Tumors
}

\author{
K.W. Yeom, L.A. Mitchell, R.M. Lober, P.D. Barnes, H. Vogel, P.G. Fisher, and M.S. Edwards
}

\begin{abstract}
BACKGROUND AND PURPOSE: Pediatric brain tumors have diverse pathologic features, which poses diagnostic challenges. Although perfusion evaluation of adult tumors is well established, hemodynamic properties are not well characterized in children. Our goal was to apply arterial spin-labeling perfusion for various pathologic types of pediatric brain tumors and evaluate the role of arterial spin-labeling in the prediction of tumor grade.
\end{abstract}

MATERIALS AND METHODS: Arterial spin-labeling perfusion of 54 children (mean age, 7.5 years; 33 boys and 21 girls) with treatment-naive brain tumors was retrospectively evaluated. The 3D pseudocontinuous spin-echo arterial spin-labeling technique was acquired at 3T MR imaging. Maximal relative tumor blood flow was obtained by use of the ROI method and was compared with tumor histologic features and grade.

RESULTS: Tumors consisted of astrocytic (20), embryonal (11), ependymal (3), mixed neuronal-glial (8), choroid plexus (5), craniopharyngioma (4), and other pathologic types (3). The maximal relative tumor blood flow of high-grade tumors (grades III and IV) was significantly higher than that of low-grade tumors (grades I and II) $(P<.001)$. There was a wider relative tumor blood flow range among high-grade tumors $(2.14 \pm 1.78)$ compared with low-grade tumors $(0.60 \pm 0.29)(P<.001)$. Across the cohort, relative tumor blood flow did not distinguish individual histology; however, among posterior fossa tumors, relative tumor blood flow was significantly higher for medulloblastoma compared with pilocytic astrocytoma $(P=.014)$.

CONCLUSIONS: Characteristic arterial spin-labeling perfusion patterns were seen among diverse pathologic types of brain tumors in children. Arterial spin-labeling perfusion can be used to distinguish high-grade and low-grade tumors.

ABBREVIATIONS: ASL = arterial spin-labeling; DIPG = diffuse intrinsic pontine glioma; DNT = dysembryoplastic tumor; OPG = optic pathway glioma; $r$ TBF $=$ relative tumor blood flow; WHO $=$ World Health Organization

B rain tumors are the most common solid tumors of childhood and are a leading cause of cancer deaths in children. ${ }^{1}$ Unlike in adults, these tumors predominate in the posterior fossa and have more heterogeneous pathologic features, including embryonal and mixed neuronal-glial types. ${ }^{2}$ Because of their diverse histologic presentation and biologic behavior, evaluation and treatment of pediatric brain tumors remain complex.

Received April 4, 2013; accepted after revision May 15.

Abstract previously presented at: Annual Meeting of the American Society of Neuroradiology, April 2012; New York, New York.

From the Departments of Radiology (K.W.Y., L.A.M., P.D.B.) Neurosurgery (R.M.L., M.S.E.), Pathology (H.V.), and Neurology (P.G.F.), Lucile Packard Children's Hospital, Stanford University, Palo Alto, California.

Please address correspondence to Kristen W. Yeom, MD, Lucile Packard Children's Hospital, Stanford University, Department of Radiology, Pediatric MRI \& CT, Room 0511, 725 Welch Rd, Palo Alto, CA 94304; e-mail: kyeom@stanford.edu

Indicates article with supplemental on-line figures.

http://dx.doi.org/10.3174/ajnr.A3670
MR imaging is important in tumor diagnosis, surgical guidance, and therapeutic monitoring of brain tumors. Various PWI methods have shown clinical usefulness in adult glioma, including the use of relative maximal CBV and relative CBF from DSCPWI to predict tumor grade and behavior. ${ }^{3-10}$ Despite abundant literature on adult brain tumors, few perfusion reports exist for pediatric brain tumors, ${ }^{11-14}$ potentially because of technical challenges. For example, the most widely available $\mathrm{T} 2^{*}$-weighted DSC imaging often requires constant, high-flow contrast injection by power injectors, double-dosing, and large-bore intravenous access, which pose challenges in young children and infants.

Recent studies have shown that arterial spin-labeling (ASL) may be a reliable alternative to DSC-PWI in the evaluation of tumor perfusion ${ }^{15-20}$ and can predict adult glioma grade. ${ }^{16}$ ASL has distinct advantages in children because of lack of contrast requirement, high SNR, labeling efficiency, and the potential for CBF quantification. Also, ASL can be repeated in cases of failed sedation or patient motion, a frequent problem in children with brain tumors. 
Although ASL has become increasingly available clinically, no ASL data exist on pediatric brain tumors. Our goal was to apply ASL perfusion for diverse pathologic types of pediatric brain tumors and evaluate the use of ASL perfusion for the prediction of tumor grade in children.

\section{MATERIALS AND METHODS \\ Patients}

A total of 54 consecutive patients younger than 19 years old with new primary brain tumors at our children's hospital were retrospectively reviewed after approval by the institutional review board (IRB-23336). The following inclusion criteria were used: patients obtained ASL at 3T as part of routine MR imaging; had no prior surgical resection, biopsy, or treatment of the tumor; underwent subsequent resection or biopsy of the tumor except in cases of optic pathway glioma (OPG) and diffuse intrinsic pontine glioma (DIPG), where tissue sampling was not used for diagnosis as part of standard care; and the solid component of the tumor exclusive of cyst, hemorrhage, or necrosis was greater than $1 \mathrm{~mL}$. Patients with underlying cardiac disease, hypertension, or vasculopathy that could alter ASL perfusion, as well as patients with motion artifacts or dental braces or hardware that degrade ASL imaging were excluded.

\section{Imaging Methods}

All patients were examined at 3T MR imaging (Discovery 750; GE Healthcare, Milwaukee, Wisconsin) by use of an 8-channel head coil. The technique used to perform perfusion ASL has been detailed elsewhere. ${ }^{21}$ In brief, ASL was performed by use of a pseudocontinuous labeling period of $1500 \mathrm{~ms}$, followed by a 1500 -ms postlabel delay. Whole-brain images were obtained with a 3D background-suppressed FSE stack-of-spirals method, with a TR of approximately 5 seconds. Multiarm spiral imaging was used, with 8 arms and 512 points acquired on each arm (bandwidth, $62.5 \mathrm{kHz}$ ), yielding in-plane and through-plane spatial resolution of 3 and $4 \mathrm{~mm}$, respectively. A high level of background suppression was achieved by use of 4 separate inversion pulses spaced around the pseudocontinuous labeling pulse. The sequence required 5 minutes to acquire, including proton attenuation images for CBF quantitation. An effort was made to position the patients' heads in the exact same position to minimize any head tilting; all scans were performed in the axial plane. For graphic prescription of the ASL, the sagittal image following the 3-plane localizer was used for alignment. Using the microsphere methodology described by Buxton et al, ${ }^{22}$ we performed postprocessing by using an automated reconstruction script that returned CBF images directly to the scanner console. Other ASL parameters were TR, $4632 \mathrm{~ms}$; TE, $10.5 \mathrm{~ms}$; FOV, $24 \mathrm{~cm} \mathrm{x} 24 \mathrm{~cm}$; matrix, $512 \times 8$; and NEX, 3 .

\section{Imaging Analysis}

For each tumor, ROIs were drawn around both the enhancing and nonenhancing solid portions of the tumor at all available axial levels of the ASL tumor blood flow map. Conventional MR imaging, including T2WI and contrast-enhanced T1WI, was used to cross-reference solid portions of the tumor to the ASL tumor blood flow maps. Areas of cyst, necrosis, and gross hemorrhage (detected by the $\mathrm{T} 2{ }^{\star}$ gradient-recalled echo and noncontrast T1WI) were avoided. A board-certified neuroradiologist (K.W.Y.) with a Certificate of Added Qualification (7 years of experience) selected ROIs blinded to clinical and pathologic data. A second blinded board-certified neuroradiologist (P.D.B.) with a Certificate of Added Qualification (30 years of experience) independently confirmed the appropriate ROI placement.

The maximal ASL signal was recorded for each ROI of the tumor, and the 2 highest values were averaged. Maximal, rather than mean, tumor blood flow was assessed, as prior studies have used maximal blood volume or flow values to correlate glioma grade and vascularity ${ }^{4-7,9,16,23}$ and have shown that maximal relative tumor blood flow (rTBF) may be most sensitive for tumor grade. ${ }^{24}$ To correct for age-dependent and patient-dependent variations of mean cerebral perfusion, ${ }^{25}$ maximal tumor blood flow was normalized to a $150-\mathrm{mm}^{2} \mathrm{ROI}$ in the contralateral gray matter to produce the maximal rTBF. Prior tumor perfusion studies have used various brain regions, including contralateral white matter, ${ }^{6,8,9}$ gray matter, ${ }^{10,17,20,26}$ or the cerebellum ${ }^{17}$ as an internal standard. For this study, contralateral gray matter, which has a higher SNR than white matter, was chosen as a reference, similar to the technique by Noguchi et al, ${ }^{23}$ because a longer arterial transit time of the white matter underestimates the ASL signal. ${ }^{27,28}$ The control ROI in healthy gray matter was placed independently by a blinded board-certified second-year neuroradiology fellow (L.A.M.), also independently confirmed by a blinded neuroradiologist (K.W.Y.). Sample ROI placement is shown in Fig 1.

Enhancement of each tumor was graded by a blinded neuroradiologist (K.W.Y.) with the following scoring system: $0=$ no enhancement, $1=<50 \%$ enhancement of the solid tissue, $2=$ $\geq 50 \%$ enhancement of the solid tissue.

\section{Pathology Findings}

All surgical specimens were reviewed by a board-certified pediatric neuropathologist (H.V.; 30 years of experience). Tumors were categorized according to the 2007 World Health Organization (WHO) Classification of Tumors of the Central Nervous System. ${ }^{29}$ For this study, WHO grade III and IV tumors were considered high grade; WHO grades I and II were considered low grade.

\section{Statistics}

We performed statistical analyses by using SPSS version 21 (IBM, Armonk, New York) with an a priori significance level $\alpha=.05$. The Kruskal-Wallis test for independent samples was used for comparison of the distribution of maximal rTBF and range between multiple tumor grades (low, high, or unspecified), specific histologic types within those grades, different posterior fossa tumors, and different locations for a given tumor type. A separate analysis of posterior fossa tumors was performed to compare perfusion differences among medulloblastoma, pilocytic astrocytomas, and ependymoma (grades II and III). Comparison of values between 2 groups, such as medulloblastoma and pilocytic astrocytoma, was performed with the Mann-Whitney $U$ test for independent samples. DIPGs were excluded from the analysis because these tumors are graded as WHO II-IV and typically do not have available pathologic 

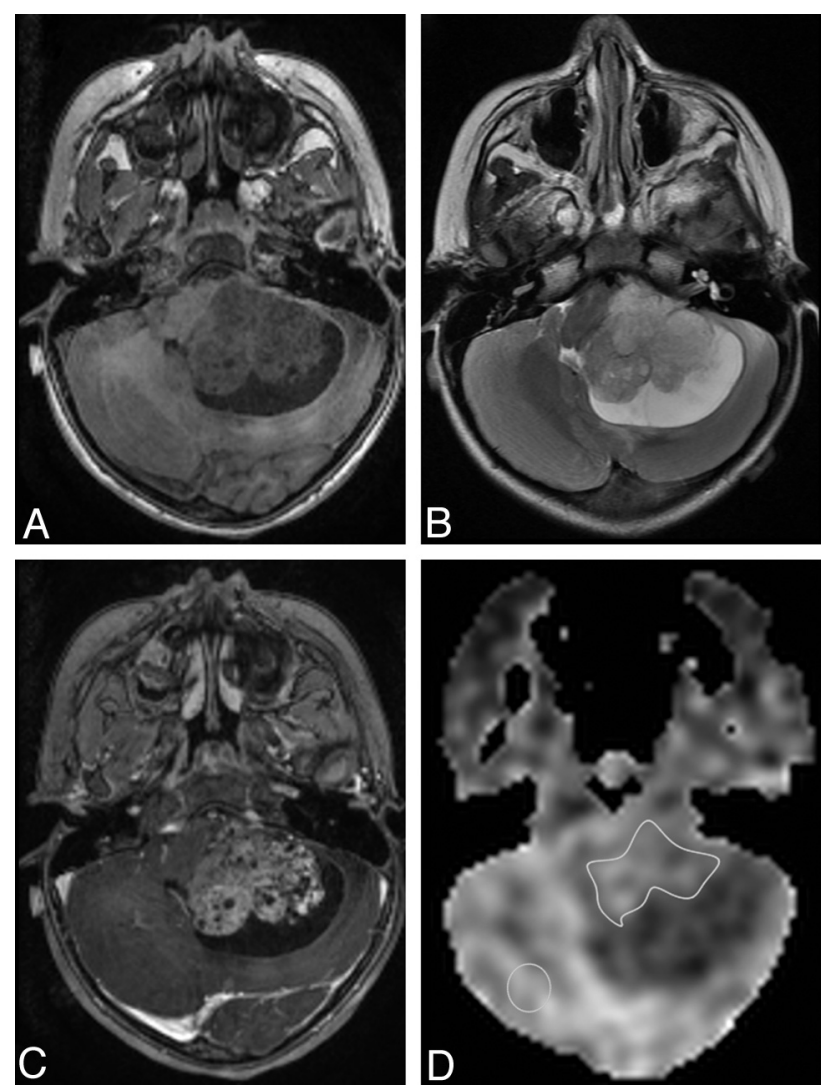

FIG 1. Example of ROI placement is shown in a 2-year-old girl with a posterior fossa atypical choroid plexus papilloma. A, Axial T1-spoiled gradient-recalled MR image. B, Axial T2-weighted MR image. C, Contrast-enhanced axial T1-spoiled gradient-recalled MR image shows enhancing mass with a cystic component. $D$, ASL perfusion map shows ROI placement over the solid tumor and in the contralateral brain not affected by the tumor.

specimens. The relationship between tumor volume and rTBF was tested by Pearson correlation.

\section{RESULTS}

\section{Patients}

The mean age of the 54 participants was 7.5 years (median age, 6 years; age range, 2 months to 18 years). There were 33 boys and 21 girls. Surgical specimens were available in 49 of these patients. Diagnoses in 4 patients with OPG and 1 patient with DIPG were based on characteristic MR imaging and clinical presentation, as these typically are not resected.

\section{Tumor Pathologic Features}

The tumors included 21 high-grade tumors (WHO III and IV), 32 low-grade tumors (WHO I and II), and 1 DIPG with unspecified grade. Tumor pathologic features by WHO grade, age, location, and rTBF are shown in the Table. Although OPGs are not typically surgically sampled, prior studies have shown that they usually represent grade I pilocytic astrocytoma ${ }^{30}$ and are therefore considered low grade.

\section{Comparison of High-Grade vs Low-Grade Tumors}

The values of averaged maximal rTBF by tumor pathologic specimen are shown in the Table. Both the maximal and averaged maximal rTBF of the high-grade tumors (grades III and IV) were significantly higher vs low-grade tumors (grades I and II) $(P<$ .001) (Fig 2). There was a wider range of rTBF (minimal to maximal rTBF) among high-grade tumors (2.14 \pm 1.78 ; median, 1.23; range, 0.55-6.96) compared with low-grade tumors ( $0.60 \pm 0.29$; median, 0.54; range, $0.32-1.66)(P<.001)$.

\section{High-Grade Tumors}

Among high-grade tumors, individual histologic features were not distinguished by $\operatorname{rTBF}(P=.377)$. The rTBF of glioblastoma $(3.70 \pm 1.89)$ was not distinguished from medulloblastoma $(2.87 \pm 1.74)$ or combined embryonal tumors (medulloblastoma, primitive neuroectodermal tumor, atypical teratoid rhabdoid tumor) $(2.62 \pm 1.66)(P=.283$ and $P=.142$, respectively). The averaged maximal rTBF ranged from $2.43-6.49$ for pediatric glioblastoma and $0.98-4.97$ for medulloblastoma. Perfusion examples are shown in Fig 3.

\section{Low-Grade Tumors}

Among low-grade tumors, individual histologic features were not distinguished by $\operatorname{rTBF}(P=.179)$. Their maximal rTBF was similar to contralateral gray matter (range, $0.89-1.12$ ). Pilocytic astrocytoma $(1.05 \pm 0.18)$ most closely approximated contralateral normal brain CBF, whereas dysembryoplastic tumors (DNTs) $(0.89 \pm 0.13)$ and OPG $(0.80 \pm 0.09)$ were hypoperfused. There was no difference in rTBF among pilocytic astrocytomas by location (cerebrum, brain stem, cerebellum) $(P=.670)$. Perfusion examples are shown in Fig 4.

\section{Posterior Fossa Tumors}

There were 22 tumors after exclusion of DIPG, consisting of medulloblastoma, ${ }^{8}$ pilocytic astrocytoma, ${ }^{7}$ ependymoma, ${ }^{3}$ choroid plexus papilloma, ${ }^{3}$ and ganglioglioma. ${ }^{1}$ Perfusion examples are shown in On-line Fig 1. Among all posterior fossa tumors, rTBF did not reliably distinguish individual histologic features $(P=$ .202). However, there was a significant difference in rTBF between medulloblastoma and pilocytic astrocytoma when compared without consideration of other tumor types (independentsampled Mann-Whitney $U$ test; $P=.014$ ). There was also a wider range of rTBF variability among medulloblastoma compared with pilocytic tumors $(P=.025)$ (On-line Fig 2$)$.

\section{Tumor Volume and Enhancement}

There was no correlation between tumor volume and $\operatorname{rTBF}(P=$ .459). The rTBF values were not different based on patterns of contrast enhancement $(P=.245)$.

\section{DISCUSSION}

Although abundant perfusion data exist in adult glioma regarding tumor detection, pathologic correlates, and therapeutic response, few studies have examined tumor perfusion in children. However, it may not always be feasible or appropriate to apply adult glioma data in the evaluation of pediatric tumors because of features unique to the pediatric population. ${ }^{31-33}$ For example, embryonal tumors, such as medulloblastoma and primitive neuroectodermal tumor, are rarely seen in adults; epidermal growth factor receptor amplification and deletion of phosphate and tensin homolog 


\begin{tabular}{|c|c|c|c|c|}
\hline Tumor Pathologic Feature & WHO Grade & Mean Age $^{\mathrm{a}}$ & Tumor Location & rTBF $^{b}$ \\
\hline \multicolumn{5}{|l|}{ High-grade tumors (III and IV) $(n=21)$} \\
\hline Glioblastoma (4) & IV & $13 \pm 8.5$ & Cerebrum & $3.70 \pm 1.89$ \\
\hline Anaplastic astrocytoma (1) & III & 11 & Cerebrum & 3.60 \\
\hline CNS PNET (1) & IV & 9 & Thalamus & 1.32 \\
\hline Medulloblastoma (9) & IV & $6.1 \pm 3.7$ & $\mathrm{PF}$ & $2.87 \pm 1.74$ \\
\hline Primary malignant melanoma (1) & IV & 12 & Cerebrum & 1.70 \\
\hline Anaplastic ependymoma (2) & III & $3 \pm 2.8$ & $\mathrm{PF}$ & $1.99 \pm 0.39$ \\
\hline CNS ATRT (1) & IV & 4 & Cerebrum & 1.67 \\
\hline Choroid plexus carcinoma (1) & III & 3 months & Lateral ventricle & 7.96 \\
\hline DIPG (1) & III & 7 & Pons & 1.70 \\
\hline \multicolumn{5}{|l|}{ Low-grade tumors (I and II) $(n=32)$} \\
\hline Pilocytic astrocytoma (8) & 1 & $9.4 \pm 6.1$ & Cerebrum (1), cerebellum (3), brain stem (4) & $1.05 \pm 0.19$ \\
\hline Hypothalamic astrocytoma (1) & II & 10 & Hypothalamus-third ventricle & 0.99 \\
\hline Angiocentric glioma (1) & I & 15 & Cerebrum & 0.94 \\
\hline DNT (2) & I & $5.5 \pm 4.9$ & Cerebrum & $0.89 \pm 0.13$ \\
\hline OPG (4) & I & $4.8 \pm 1.5$ & Optic chiasm & $0.80 \pm 0.09$ \\
\hline Choroid plexus papilloma (3) & I & $5.6 \pm 3.5$ & $\operatorname{PF}(2)$, lateral ventricle (1) & $1.13 \pm 0.15$ \\
\hline Atypical choroid plexus papilloma (1) & II & 2 & $\mathrm{PF}$ & 1.34 \\
\hline Ganglioglioma (6) & I & $6.2 \pm 6.6$ & Cerebrum (5), brain stem (1) & $1.45 \pm 0.54$ \\
\hline Craniopharyngioma (4) & I & $12.8 \pm 4.3$ & Suprasellar cistern & $0.99 \pm 0.13$ \\
\hline Atypical meningioma (1) & II & 8 months & Cerebrum & 1.60 \\
\hline Ependymoma (1) & II & 3 & $\mathrm{PF}$ & 1.82 \\
\hline \multicolumn{5}{|l|}{ Unspecified grade $(n=1)$} \\
\hline DIPG (1) & II-IV & 6 & Pons & 1.35 \\
\hline
\end{tabular}

Note:-ATRT indicates atypical teratoid rhabdoid tumor; PF, posterior fossa; PNET, primitive neuroectodermal tumor.

${ }^{a}$ Age in years, unless otherwise specified.

${ }^{b}$ rTBF equals the averaged maximal rTBF values from 2 different ROIs within the solid tumor.

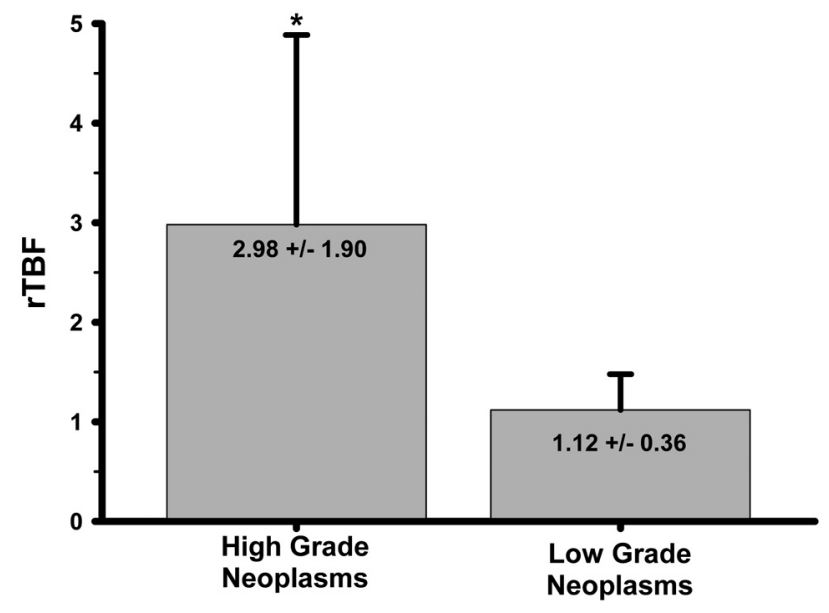

FIG 2. A bar graph showing comparison of high-grade and low-grade neoplasms. Significantly higher mean $\operatorname{rTBF}\left({ }^{*}\right)$ is seen in high-grade compared with low-grade tumors. The error bars represent standard deviation.

gene, commonly found in adult primary glioblastoma, are rarely detected in pediatric glioblastoma. ${ }^{31,33}$ With increased use of antiangiogenic therapies, including treatment of pediatric highgrade astrocytomas and treatment-resistant medulloblastoma and pilocytic astrocytoma, PWI may be a key to tumor surveillance and identification of treatment-responsive tumor subtypes.

The benefits of ASL perfusion in children are well known, including high labeling efficiency, SNR, and lack of contrast requirement. ${ }^{34}$ Immature paranasal sinuses also likely contribute to improved image quality of ASL, with reduced distortion artifacts in the frontal and inferior brain regions in young children. ${ }^{35}$ Here, we used spin-echo ASL that incorporates high-field parallel imaging, pseudocontinuous labeling, and 3D imaging with back- ground suppression, which has previously been shown to increase sensitivity for CBF imaging. ${ }^{36}$ To our knowledge, our study is the first to report ASL parameters of various pathologic types of pediatric brain tumors and its use in correlating tumor grade in children.

Our results for neuronal-glial and astrocytic tumors are similar to nuclear medicine and adult perfusion studies. Similar to a prior report by Kumabe et $\mathrm{al}^{37}$ that showed high uptake in their 3 cases of ganglioglioma by use of thallium-201 SPECT, tumor blood flow was elevated compared with gray matter in 5 of 6 gangliogliomas in our study. In contrast, DNTs, which generally have low growth potential, ${ }^{38}$ showed lower rTBF, similar to hypoperfusion pattern by use of iodine $123 \mathrm{~N}$-isopropyl- $p$-iodoamphetamine or technetium Tc99m hexamethylpropyleneamine oxime methods. ${ }^{39}$ In our study, rTBF for glioblastoma ranged from 2.43-6.49, similar to the wide range of 1.18-5.86 reported for adult high-grade gliomas. ${ }^{16,24}$ Also, rTBF of $0.78-1.37$ for grade I/II glioma was similar to the $0.59-0.96$ range reported for adult low-grade gliomas. ${ }^{16,24}$ Different ASL techniques and reference brain regions may account for some variations. A wide rTBF range for high-grade neoplasms suggests vascular heterogeneity, which also has been noted for adult glioblastoma by use of DSC perfusion. ${ }^{9}$ The ability of ASL maps to depict tumor vascular heterogeneity and indicate higher tumor blood flow regions offers a useful parameter, which could help direct biopsy of higher vascular density or more malignant regions.

Pilocytic astrocytomas are typically considered vascular with characteristic hyalinized and glomerular vessel formation along the cyst wall. ${ }^{40}$ In our study, rTBF of pilocytic astrocytoma was not elevated and was similar to contralateral gray matter. This finding may be attributed to the characteristic capillary exchange rate within the pilocytic tumor tissues, vessel density, and micro- 

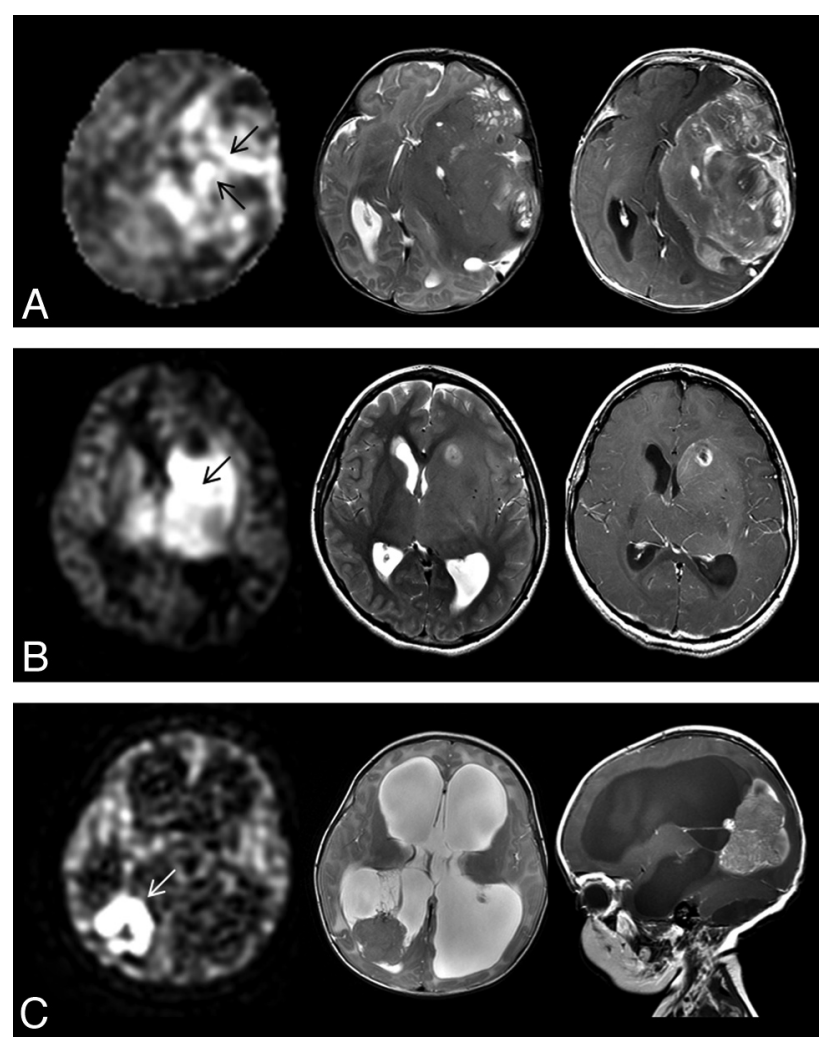

FIG 3. ASL perfusion (left) of various high-grade tumors and correlative axial T2-weighted MR image (middle) and axial contrast-enhanced T1-weighted MR image (right). A, Heterogeneous signal with regions of high rTBF (arrows) is seen in a 2-month-old girl with a large hemispheric glioblastoma. B, High rTBF signal (arrow) is seen in an 11-year-old girl. Biopsy of tumor just posterior to the necrosis showed anaplastic astrocytoma histology. Given the presence of necrosis, this was considered mixed anaplastic astrocytoma-glioblastoma. C, Markedly high rTBF (arrow) is seen within choroid plexus carcinoma in a 3-month-old girl.

vascular architecture or integrity. ${ }^{41,42}$ Although not statistically different, OPG consistently showed slightly lower perfusion than other pilocytic astrocytomas. Given reports of increased microvessel density in OPG with more aggressive behavior, ${ }^{43}$ exclusion of OPG that required therapy could have biased toward a subset of less vascular, or biologically less aggressive, OPG in our cohort.

Although posterior fossa tumors comprise approximately $50 \%$ of pediatric brain tumors, ${ }^{44}$ perfusion parameters of these tumors are relatively unknown, in part, because of general technical challenges of DSC perfusion requiring large intravenous access or power injection and posterior fossa susceptibility artifacts when using the gradient-echo technique. Here, we showed higher perfusion in medulloblastoma compared with pilocytic astrocytoma, which may complement DWI in distinguishing these tumors, as previously described by Rumboldt et al. ${ }^{45}$ Although medulloblastoma, on average, showed higher rTBF than ependymoma, overlap between these 2 tumors was seen because of wide perfusion variability of medulloblastoma.

Such heterogeneous perfusion among medulloblastoma is noteworthy. Although medulloblastomas are classically categorized based on the presence of densely packed round cells, pathologic "variants" (large-cell or anaplastic medulloblastoma,
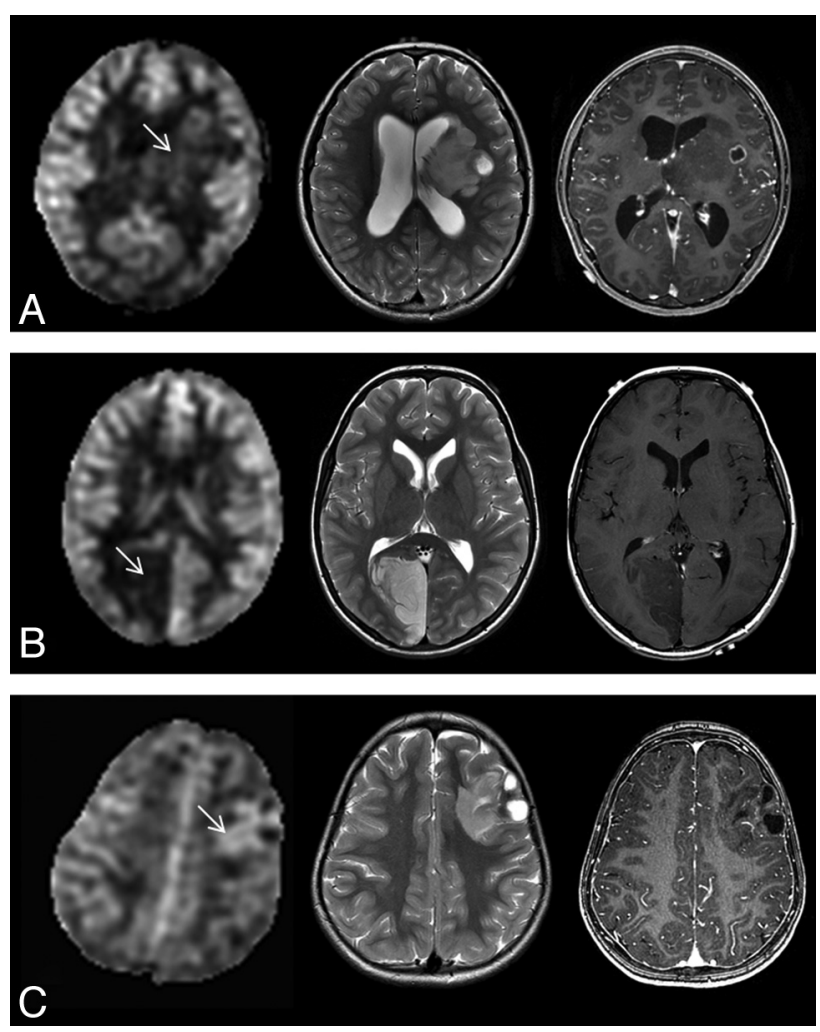

FIG 4. ASL perfusion (left) of various low-grade tumors and correlative axial T2-weighted MR image (middle) and axial contrast-enhanced T1-weighted MR image (right). A, Low rTBF is seen within the pilocytic astrocytoma (arrow) with some tumor regions that show ASL signal similar to the contralateral gray matter in a 9-year-old boy. $B$, DNT shows low ASL signal (arrow) in a 9-year-old boy. C, Higher rTBF (arrow) compared with DNT (B) is seen in a 3-year-old girl with left frontal ganglioglioma.

desmoplastic medulloblastoma, and medulloblastoma with extensive nodularity) with unique histologic features and biologic or clinical behavior are now recognized and incorporated in the 2007 WHO classification. Investigators have further shown heterogeneous imaging features ${ }^{46}$ and marked genetic diversity of medulloblastoma with at least 4 unique molecular subgroups (WNT, SHH, group C, and group D) having distinct transcription profiles, chromosomal aberrations, and clinical behavior. ${ }^{47,48}$ Of note, 4 of the 8 medulloblastomas with the highest rTBF (range, 2.16-4.97) demonstrated the most aggressive behavior, with death occurring within 11 months of tumor diagnosis in 3 patients and the remaining patient classified as having high-risk disease at the time of the study because of leptomeningeal seeding. Further studies are needed to determine perfusion and genomic correlates and the potential prognostic role of perfusion imaging in pediatric medulloblastoma.

We observed no significant difference in rTBF values on the basis of the degree of contrast enhancement. Perfusion measurements of tumors by ASL are much less sensitive to permeability changes than the DSC methods, which are more affected by impaired blood-brain barrier function. ${ }^{24,49}$ It is likely that the ASL signal of our tumors represented tumor perfusion on the basis of vascular density as previously described by Noguchi et al. ${ }^{23}$

We recognized a few limitations in our study. Although most of the patients presented with headaches, nausea or vomiting, and 
focal neurologic deficits, a small number of those with seizures, specifically those with ganglioglioma or DNTs, could have presented with altered brain hemodynamics. ${ }^{50}$ Elevated intracranial pressure from brain tumor could potentially reduce global cerebral perfusion and thereby decrease SNR and accuracy of the CBF and tumor blood flow measurements. To mitigate this problem, as well as age and other patient-dependent factors that could affect cerebral perfusion, tumor blood flow was referenced to normal brain as an internal control. Also, given general unknown effects of sedatives on ASL imaging, sedation status remains an important consideration in pediatric imaging. ${ }^{49}$ However, for relative ASL tumor blood flow measures, its effect is likely small because of its focus on regional hemodynamic change, similar to presumed insignificant effect on relative calculations obtained by the DSC method. ${ }^{49}$ Prior investigators have shown that ASL underestimates $\mathrm{CBF}$ in brain regions with delayed flow, such as the white matter. ${ }^{27}$ It is possible that ASL could underestimate tumor blood flow in cases of tortuous vasculature from angiogenesis because of delay in signal arrival, and alternatively, overestimate tumor blood flow in cases of vascular shunting. Despite reports of fewer susceptibility artifacts compared with gradient-echo PWI, ${ }^{17}$ many patients were excluded from our study because of dental braces, which rendered the ASL nondiagnostic, an important factor in clinical implementation.

\section{CONCLUSIONS}

Characteristic ASL perfusion patterns were seen among diverse pathologic types of brain tumors in children and can be used to distinguish high-grade and low-grade tumors.

ICMJE Disclosures: Michael Edwards-UNRELATED: Grants/Grants Pending: Stanford University, ${ }^{*}$ Comments: Private funding from donors as well as National Institutes of Health and Brain Tumor Funding Grant Associations.

\section{REFERENCES}

1. Gurney JG, Smith MA, Bunin GR. CNS and miscellaneous intracranial and intraspinal neoplasms. In: Ries L, Smith M, Gurney J, eds. Cancer Incidence and Survival Among Children and Adolescents: United States SEER Program 1965-1995. NIH Publication No. 994649. Bethesda, Maryland: National Cancer Institute SEER Program;1999:51-63

2. Pollack IF. Brain tumors in children. $N$ Engl J Med 1994; 331:1500-07

3. Sugahara T, Korogi Y, Kochi M, et al. Correlation of MR imagingdetermined cerebral blood volume maps with histologic and angiographic determination of vascularity of gliomas. AJR Am J Roentgenol 1998;171:1479-86

4. Knopp EA, Cha S, Johnson G, et al. Glial neoplasms: dynamic contrast-enhanced T2*-weighted MR imaging. Radiology 1999;211: 791-98

5. Sugahara T, Korogi Y, Kochi M, et al. Perfusion-sensitive MR imaging of gliomas: comparison between gradient-echo and spin-echo echo-planar imaging techniques. AJNR Am J Neuroradiol 2001; 22:1306-15

6. Shin JH, Lee HK, Kwun BD, et al. Using relative cerebral blood flow and volume to evaluate the histopathologic grade of cerebral gliomas: preliminary results. AJR Am J Roentgenol 2002;179:783-89

7. Law M, Yang S, Wang H, et al. Glioma grading: sensitivity, specificity, and predictive values of perfusion MR imaging and proton MR spectroscopic imaging compared with conventional MR imaging. AJNR Am J Neuroradiol 2003;24:1989-98

8. Law M, Yang S, Babb JS, et al. Comparison of cerebral blood volume and vascular permeability from dynamic susceptibility contrastenhanced perfusion MR imaging with glioma grade. AJNR Am J Neuroradiol 2004;25:746-55

9. Aronen HJ, Gazit IE, Louis DN, et al. Cerebral blood volume maps of gliomas: comparison with tumor grade and histologic findings. $\mathrm{Ra}$ diology 1994;191:41-51

10. Law M, Oh S, Babb JS, et al. Low-grade gliomas: dynamic susceptibility-weighted contrast-enhanced perfusion MR imagingprediction of patient clinical response. Radiology 2006;238:658-67

11. Tzika AA, Astrakas LG, Zarifi MK, et al. Spectroscopic and perfusion magnetic resonance imaging predictors of progression in pediatric brain tumors. Cancer 2004;100:1246-56

12. Cha S. Dynamic susceptibility-weighted contrast-enhanced perfusion MR imaging in pediatric patients. Neuroimaging Clin N Am 2006;16:137-47, ix

13. Thompson EM, Guillaume DJ, Dósa E, et al. Dual contrast perfusion MRI in a single imaging session for assessment of pediatric brain tumors. J Neurooncol 2012;109:105-14

14. Löbel U, Sedlacik J, Reddick WE, et al. Quantitative diffusionweighted and dynamic susceptibility-weighted contrast-enhanced perfusion MR imaging analysis of T2 hypointense lesion components in pediatric diffuse intrinsic pontine glioma. AJNR Am J Neuroradiol 2011;32:315-22

15. Kimura H, Takeuchi H, Koshimoto $Y$, et al. Perfusion imaging of meningioma by using continuous arterial spin labeling: comparison with dynamic susceptibility-weighted contrast-enhanced MR images and histopathologic features. AJNR Am J Neuroradiol 2006;27:85-93

16. Warmuth C, Gunther M, Zimmer C. Quantification of blood flow in brain tumors: comparison of arterial spin labeling and dynamic susceptibility-weighted contrast-enhanced MR imaging. Radiology 2003;228:523-32

17. Järnum H, Steffensen EG, Knutsson L, et al. Perfusion MRI of brain tumors: a comparative study of pseudo-continuous arterial spin labeling and dynamic susceptibility contrast imaging. Neuroradiology 2010;52:307-17

18. White CM, Pope WB, Zaw T, et al. Regional and voxel-wise comparisons of blood flow measurements between DSC magnetic resonance imaging (DSC-MRI) and arterial spin labeling (ASL) in brain tumors. J Neuroimaging 2014;24:23-30

19. van Westen D, Petersen ET, Wirestam R, et al. Correlation between arterial blood volume obtained by arterial spin labeling and cerebral blood volume in intracranial tumours. MAGMA 2011;24:211-23

20. Hirai T, Kitajima M, Nakmura H., et al. Quantitative blood flow measurements in gliomas using arterial spin labeling at 3T: intermodality agreement and inter- and intraobserver reproducibility study. AJNR Am J Neuroradiol 2011;32:2073-79

21. Dai W, Garcia D, Bazelaire C, et al. Continuous flow-driven inversion for arterial spin labeling using pulsed radio frequency and gradient fields. Magn Reson Med 2008;60:1488-97

22. Buxton RB, Frank LR, Wong EC, et al. A general kinetic model for quantitative perfusion imaging with arterial spin labeling. Magn Reson Med 1998;40:383-96

23. Noguchi T, Yshiura T, Hiwatashi A. Perfusion imaging of brain tumors using arterial spin labeling: correlation with histopathologic vascular density. AJNR Am J Neuroradiol 2008;29:688-93

24. Wolf RL, Wang J, Wang S, et al. Grading of CNS neoplasms using continuous arterial spin labeled perfusion MR imaging at 3 Tesla. J Magn Reson Imaging 2005;22:475-82

25. Parkes LM, Rashid W, Chard DT, et al. Normal cerebral perfusion measurements using arterial spin labeling: reproducibility, stability, and age and gender effects. Magn Reson Med 2004;51:736-43

26. Ulmer S, Helle M, Jansen O, et al. Intraoperative dynamic susceptibility contrast weighed magnetic resonance imaging (iDSC-MRI)technical considerations and feasibility. Neuroimage 2009;45:38-43 27. van Gelderen P, de Awart JA, Duyn JH. Pitfalls of MRI measure- 
ments of white matter perfusion based on arterial spin labeling. Magn Reson Med 2008;59:788-95

28. Ye FQ, Berman KF, Ellmore T, et al. H2 150 PET validation of steady-state arterial spin tagging cerebral blood flow measurements in humans. Magn Reson Med 2000;44:450-56

29. Louis DN, Ohgaki H, Wiestler OD, et al, eds. WHO Classification of Tumours of the Central Nervous System, Fourth Edition. Lyon, France: International Agency for Research on Cancer; 2007

30. Kleihues P, Cavenee WK, eds. World Health Organization Classification of Tumours: Pathology and Genetics of Tumours of the Nervous System. Lyon, France: International Agency for Research on Cancer; 2000

31. Bredel M, Pollack IF, Hamilton RL, et al. Epidermal growth factor receptor (EGFR) expression and gene amplification in high grade non-brainstem gliomas of childhood. Clin Cancer Res 1999;5: 1786-92

32. Rickert CH, Strater R, Kaatsch P, et al. Pediatric high-grade astrocytomas show chromosomal imbalances distinct from adult cases. Am J Pathol 2001;158:1525-32

33. Suri V, Das P, Jain A, et al. Pediatric glioblastoma: a histopathological and molecular genetic study. Neuro Oncol 2009;11:274-80

34. Wang J, Licht DJ, Jahng GH, et al. Pediatric perfusion imaging using pulsed arterial spin labeling. J Magn Reson Imaging 2003;18:404-13

35. Deibler AR, Pollock JM, Kraft RA, et al. Arterial spin-labeling in routine clinical practice, part 1: technique and artifacts. AJNR Am J Neuroradiol 2008;29:1228-34

36. Wolf RL, Detre JA. Clinical neuroimaging using arterial spin-labeled perfusion magnetic resonance imaging. Neurotherapeutics 2007;4:346-59

37. Kumabe T, Shimazu H, Sonoda $Y$, et al. Thallium-201 single-photon emission computed tomographic and proton magnetic resonance spectroscopic characteristics of intracranial ganglioglioma: three technical case reports. Neurosurgery 1999;45:183-187; discussion 187

38. Daumas-Duport C, Pietsch T, Lantos PL. Dysembryoplastic neuroepithelial tumour. In: Kleihues P, Cavenee WK, eds. World Health Organization Classification of Tumours: Pathology and Genetics of Tumours of the Nervous System. Lyon, France: International Agency for Research on Cancer; 2000:103-106
39. Abe M, Tabuchi K, Tsuji T, et al. Dysembryoplastic neuroepithelial tumor: report of three cases. Surg Neurol 1995;43:240-245

40. Burger PC, Scheithauer BW, Paulus W, et al. Pilocytic astrocytoma. In: Kleihues P, Cavenee W, eds. World Health Organization Classification of Tumours: Pathology and Genetics of Tumours of the Nervous System. Lyon, France: International Agency for Research on Cancer; 2000:45-51

41. Birlik, Canda S, Ozer E. Tumour vascularity is of prognostic significance in adult, but not paediatric astrocytomas. Neuropathol Appl Neurobiol 2006;32: 532-38

42. Sie M, de Bont ES, Scherpen FJ, et al. Tumour vasculature and angiogenic profile of paediatric pilocytic astrocytoma; is it much different from glioblastoma? Neuropathol Appl Neurobiol 2010;36: $636-47$

43. Bartels U, Hawkins C, Jing M, et al. Vascularity and angiogenesis as predictors of growth in optic pathway/hypothalamic gliomas. J Neurosurg 2006;104:314-20

44. Centers for Disease Control and Prevention. Data Collection of Primary Central Nervous System Tumors. National Program of Cancer Registries Training Materials. Atlanta, Georgia: Department of Health and Human Services, Centers for Disease Control and Prevention; 2004

45. Rumboldt Z, Camacho DL, Lake D, et al. Apparent diffusion coefficients for differentiation of cerebellar tumors in children. AJNR Am J Neuroradiol 2006;27:1362-69

46. Yeom KW, Mobley BC, Lober RM, et al. Distinctive MRI features of pediatric medulloblastoma subtypes. $A J R A m J$ Roentgenol 2013;200:895-903

47. Northcott PA, Korshunov A, Witt H, et al. Medulloblastoma comprises four distinct molecular variants. J Clin Oncol 2011;29: $1408-14$

48. Cho YJ, Tsherniak A, Tamayo P, et al. Integrative genomic analysis of medulloblastoma identifies a molecular subgroup that derives poor clinical outcome. J Clin Oncol 2011;29:1424-30

49. Wang J, Licht DJ. Pediatric perfusion MR imaging using arterial spin labeling. Neuroimaging Clin N Am 2006;16:149-67, ix

50. Toledo M, Munuera J, Salas-Puig X, et al. Localisation value of ictal arterial spin-labelled sequences in partial seizures. Epileptic Disord 2011;13:336-39 